\title{
CENTRO DE CONVIVÊNCIA PARA IDOSOS EM TEMPOS DE PANDEMIA: ESTRATÉGIAS DE ACOMPANHAMENTO A DISTÂNCIA
}

\section{Beatriz Lopes Rezende Nunes' ORCID: 0000-003-3304-378X}

Anna Beatriz Rodrigues Santos Ferreira' ORCID: 0000-0001-7942-572X

Carla Silva de Andrade' ORCID: 0000-0001-8562-7766

Letícia Oppenheimer Cury' ORCID: 0000-0003-4708-1584

Graziele Ribeiro Bitencourt" ORCID: 0000-0002-9130-9307

Priscilla Alfradique de Souza' ORCID: 0000-0002-4625-7552

'Universidade Federal do Estado do Rio de Janeiro. Rio de Janeiro, Rio de Janeiro, Brasil.

"Universidade Federal Rio de Janeiro. Macaé, Rio de Janeiro, Brasil.

Autor Correspondente: Beatriz Lopes Rezende Nunes E-mail: beatrizlrn1@edu.unirio.br

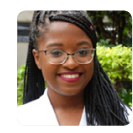

Como citar:

Nunes BLR, Ferreira ABR, Andrade CS, Cury LO, Bitencourt GR, Souza PA. Centro de convivência para idosos em tempos de pandemia: estratégias de acompanhamento a distância. In: Santana RF (Org.). Enfermagem gerontologica no cuidado do idoso em tempos da COVID 19. 2.ed.rev. Brasilia, DF: Editora ABEn; 2020. p 36-41.

(Serie Enfermagem e Pandemias, 2). https://doi.org/10.51234/aben.20.e02.c06

\section{INTRODUÇÃO}

O novo coronavírus, denominado COVID-19, teve seu surgimento em Wuhan, China, em 31 de dezembro de 2019. Com os níveis de contaminação se elevando incessantemente, além do número de óbitos, e estendendo-se para outros continentes, a Organização Mundial da Saúde (OMS) declarou no dia 30 de janeiro de 2020, que esse evento constituía uma Emergência de Saúde Pública e Importância Internacional' ${ }^{1}$. Além disso, em consequência do alarmante crescimento do número de casos e óbitos no Irã e Itália, em 24 de fevereiro de 2020, o Ministério da Saúde (MS) alterou a definição de caso suspeito, passando a incluir pacientes que estiveram em mais 8 países, totalizando 16 países².

O cenário nacional atual aponta 29,9 milhões de idosos, tendo uma estimativa para o ano de 2100 de 72,4 milhões de pessoas nesse grupo etário. No mundo, esse panorama converge como o do Brasil, com 1,1 bilhão de idosos em 2020 , e estimativa de 3,1 bilhões em $2100^{1}$. Com o envelhecimento populacional em evidência, é notória que essa parte significativa da população necessita de maiores cuidados para manter a qualidade desse processo natural da vida. Tal necessidade também se estende para o cenário da pandemia da COVID-19, visto que muitos idosos possuem doenças crônicas e a imunossenescência os deixa vulneráveis às doenças infectocontagiosas ${ }^{3}$.

Não é por acaso que a população idosa está no centro da discussão dessa pandemia. No Brasil, a primeira vítima fatal foi um homem de 62 anos, que possuía hipertensão arterial sistêmica e diabetes mellitus. Tais doenças crônicas são associadas ao envelhecimento e também atribuídas como parte do grupo de risco para COVID-19. Nesse sentido, as evidências até o momento ratificam que a vulnerabilidade aumenta conforme a idade, apontando maior taxa de mortalidade entre idosos com 80 anos ou mais. Dentre esses, $14,8 \%$ dos infectados chegaram a óbito; enquanto entre os idosos de 60 a 79 anos tem-se uma taxa de $8,0 \%$ a $8,8 \%{ }^{1}$. 
Perante tais condições, foram propostas diversas maneiras de inibir a transmissão, sendo as principais, o distanciamento e o isolamento social, que desaceleram a propagação da doença, e proposição de redução do pico de ocorrência. Esta medida tem impactado diretamente o dia a dia de toda a população, tanto no quesito de rotinas modificadas, como em relação à saúde mental que também é afetada 4 .

Os idosos, que representam $13 \%$ da população brasileira, sofrem diretamente esse impacto, pois ao se depararem com o bloqueio das atividades e a avalanche de notícias que os colocam como o centro da atenção, e acabam por maiores chances de desenvolverem de medo e angústia 5 . Há também uma diversidade e pluralidade do envelhecimento humano, como idosos que residem sozinhos e se sentem abandonados, principalmente aqueles que não possuem contato com familiares, ou que assumem papéis de cuidadores de crianças e adolescentes. Há ainda idosos dependentes, que podem causar sobrecarga e aumento do estresse do cuidador ${ }^{3}$.

Desse modo, ações focadas na promoção da qualidade do envelhecimento populacional necessitam de visibilidade e viabilidade. Além disso, também se faz relevante a valorização da pessoa idosa, estimulando autonomia e independência. Nesse sentido, os centros de convivência para a pessoa idosa tem como enfoque atenção as questões relacionadas a promoção do envelhecimento saudável e atuam com funcionamento diário para desenvolvimento de diversas atividades, como roda de conversas com temas relevantes da atualidade, terapias, atividades físicas, oficinas de estímulo à memória e cognição, dança, dentre outras, proporcionando bem-estar físico, psíquico e emocional, através do acompanhamento pela equipe multiprofissional ${ }^{6}$.

Diante desse cenário, os profissionais da saúde também tiveram que se reorganizar e reinventar a forma do atendimento realizado. Desse modo, devido às limitações causadas pela pandemia, tem-se proposto diferentes estratégias de convivência à distância. Essas estratégias objetivam que as tecnologias consigam estabelecer um elo com as antigas atividades rotineiras e estimulem o amparo das novas emoções e sentimentos que a pandemia trouxe à realidade atual dos idosos ${ }^{6}$.

Como novas medidas de manutenção à saúde e atenção a pessoa idosa, o acompanhamento à distância que possibilita a comunicação e a interação através de ligações, os e-mails e aplicativos para smartphones, que realizam trocas de mensagens de texto instantaneamente, além de vídeos, fotos e mensagens de áudio. Nesse sentido, os profissionais de saúde têm recebido autorização dos conselhos para acompanhamento remoto durante a pandemia. Assim, os enfermeiros também tiveram sua atuação ampliada. Conforme a resolução do COFEN N ${ }^{\circ} 634 / 2020$, a teleconsulta de enfermagem permite o uso desses meios tecnológicos para combater a pandemia COVID-19. Tal recurso, além de manter o acompanhamento desse idoso, possibilita ações que visam orientar, esclarecer e encaminhar em caso de necessidade, mantendo o vínculo entre o profissional e o cliente?.

Perante tal necessidade e extrema importância do distanciamento social versus manutenção da saúde multidimensional do idoso, surge o impulso inspirador da criação de novas estratégias de convivência à distância. Como consequência, a necessidade de reflexão sobre o assunto, a fim de compreensão das possibilidades e desafios dessa nova realidade, que diz respeito a atenção ao idoso, seu contexto familiar e cultural.

\section{OBJETIVO}

Descrever a experiência vivenciada na utilização de estratégias de acompanhamento a distância em um centro de convivência para idosos em tempos de pandemia COVID-19.

\section{MÉTODO}

Trata-se de um relato de experiência do desenvolvimento de atividades à distância em um centro de convivência - Programa Interdisciplinar de Promoção à Saúde e Qualidade de Vida do Idoso - Grupo Renascer pertencente ao Hospital Universitário Gaffreé e Guinle (HUGG), da Universidade Federal do Estado do Rio de Janeiro (UNIRIO). O Grupo Renascer possui 235 idosos cadastrados. Destes, 150 participaram de atividades à 
distância pela equipe multidisciplinar: educação física, enfermagem, fisioterapia, nutrição, psicologia, serviço social, medicina e terapia ocupacional. As atividades desenvolvidas pelo Renascer são programadas e distribuídas ao longo da semana, para que assim os idosos tenham escolha e agendamento das dinâmicas que participarão.

As atividades à distância iniciaram em março de 2020, logo após a determinação das medidas de distanciamento social do MS, com o planejamento das ações e distribuição de tarefas. O segundo momento iniciou-se com os telefonemas para os idosos e envio de materiais, apostilas e cartilhas com atividades, dentre essas: "Apostila de exercício Cognitivo" e "Programa de Exercícios para idosos, durante o tempo de isolamento" e teleoficinas. Além disso, também foi compartilhado o vídeo "Cuidados da Quarentena” desenvolvido pela liga acadêmica de gerontologia da UNIRIO.

Também tem sido desenvolvida a teleconsulta de enfermagem, no qual foram atendidos até o momento, 111 idosos ao longo das semanas decorridas, através de ligações e contatos por aplicativo de mensagem instantânea. Com isso, é realizado o monitoramento dos idosos previamente acompanhados na consulta de enfermagem gerontológica presencial. Conforme necessidade e planejamento da assistência, atua-se conjuntamente com a equipe multidisciplinar. Dentro desta estratégia de teleconsulta, realiza-se o acompanhamento periódico do idoso, para avaliação de suas condições de saúde, cuidados frente a pandemia COVID-19 e promoção do envelhecimento saudável.

\section{RESULTADOS}

Com a necessidade de nova organização das atividades na modalidade de acompanhamento a distância, a equipe multiprofissional vem desenvolvendo atividades remotas, mantendo-se os grupos de atividades previamente desenvolvidas ou com o acompanhamento individual, conforme especificidade (Figura 1).

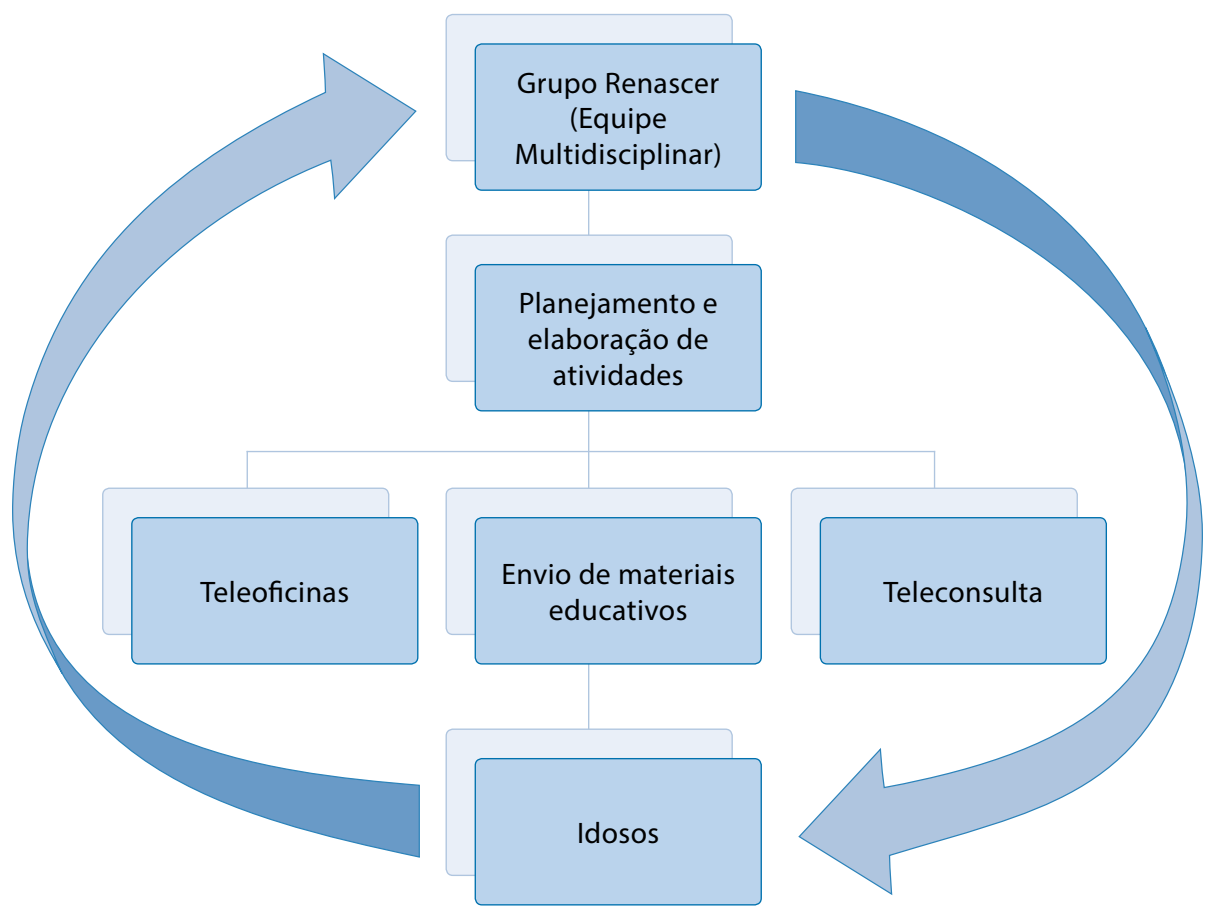

Figura 1 - Dinâmica de acompanhamento dos idosos a distância 
Foram desenvolvidos materiais para a população idosa do centro de convivência. Dentre esses, tem-se tarefas individuais, apostilas de estimulação cognitiva, motora e de atividades físicas em domicílio e vídeos com orientações sobre cuidados na pandemia da COVID-19. Associados a estes materiais, foram disponibilizados conteúdos teóricos e dicas de atividades nas redes sociais do projeto de extensão. A "Apostila de Exercícios Cognitivos", que contém mais de 100 exercícios, foi desenvolvida pela equipe multidisciplinar do Grupo Renascer, em parceria com equipe Portuguesa de gerontologia, tendo como objetivo estimular funções cognitivas associadas a atenção, percepção, estratégia, raciocínio, memória e concentração ${ }^{8}$.

A segunda apostila intitulada "Programa de Exercícios para idosos durante o tempo de isolamento", possui 10 exercícios de estimulação motora, que podem ser feitos em casa com objetos do cotidiano, facilitando o desenvolvimento de atividades físicas pelo idoso em domicílio. Outro material interessante, desenvolvido pela liga acadêmica de enfermagem em gerontologia da UNIRIO, foi um vídeo com cerca de um minuto de duração, com informações e orientações para idosos e familiares para a prevenção da Covid-199.

As ligações para distribuição dos materiais desenvolvidos para 150 idosos, foram realizadas por bolsistas do projeto de extensão. Elas foram feitas em horário comercial, de segunda a sexta-feira. Nesta ligação, eram confirmadas informações sobre os idosos e realizadas avaliações iniciais: como o idoso estava se sentindo em relação ao contexto mundial da pandemia; se ele possuía algum endereço de e-mail para contato, telefone celular com aplicativo de mensagem de texto instantânea ou alguma rede social ativa. A partir das informações referentes ao meio de comunicação preferencial, os materiais foram enviados.

Toda a equipe multiprofissional readaptou-se ao desenvolvimento de atividades remotas; a equipe de psicólogas tem realizado acompanhamento individual e em grupo. A fisioterapia também tem enviado materiais educativos aos idosos. Já a equipe médica reajustou-se com consultas online, além da manutenção do serviço de atendimento presencial para casos específicos.

Tendo em vista a continuidade do cuidado desenvolvido, foram realizadas teleconsultas de enfermagem ${ }^{7}$. A estratégia do telecuidado foi protocolada através de ferramentas disponíveis em formulários eletrônicos, que serviram como base para cada consulta realizada. Além disso, a equipe de enfermagem foi treinada para padronização do atendimento e seus fluxos. Contudo, ressalta-se que nas ligações também foram atendidas as especificidades e necessidades de cada idoso e sua família.

Toda a estratégia de telecuidado foi baseada no processo de enfermagem (PE), através da coleta de dados, nome completo, data de nascimento, idade e informações pertinentes dos idosos; identificação dos diagnósticos de enfermagem; determinação dos resultados esperados; realização de intervenções por meio de orientações de prevenção e promoção da saúde e; avaliação dos cuidados realizados ${ }^{10}$. Assim, para desenvolvimento da atenção à saúde por telecomunicação remota, faz-se necessária a manutenção das relações sociais estáveis e de confiança.

A partir de tais estratégias, o telecuidado promove a manutenção do vínculo da pessoa idosa com o centro de convivência, além da reinvenção das formas de socialização. Para que a relação seja mantida até que o retorno presencial se restabeleça, é essencial a manutenção do vínculo que esse idoso possuía outrora. Dessa forma, o telecuidado auxilia o cuidado de enfermagem e ampara o idoso no alcance de melhores resultados de maneira segura ${ }^{11}$.

A capacidade de se readaptar e propor novas estratégias de cuidar foi extremamente importante nesse contexto de distanciamento social. Além disso, as tecnologias de informação e comunicação se apresentaram como grande aliadas, ao permitir que profissionais de saúde e idosos mantivessem suas atividades e rotinas de cuidado de maneira remota.

A interdisciplinaridade da equipe tem como objetivo a manutenção e promoção da saúde. O conhecimento compartilhado é aplicado para alcançar os melhores resultados para o idoso, mesmo diante dos diferentes cenários inerentes ao envelhecimento ${ }^{12}$. Sendo assim, no cenário atual de pandemia, torna-se imprescindível trabalhar com a equipe multiprofissional em busca do olhar integral. 
Contudo, observou-se em muitos atendimentos a felicidade dos idosos em receber ligações advindas do centro de convivência no qual estão vinculados. Era perceptível o desejo de retorno às atividades o quanto antes, além da falta de comunicação com outras pessoas diferentes daquelas que fazem parte do seu núcleo familiar. Por conta disso, alguns idosos mantiveram contato semanal com a equipe de enfermagem dos projetos de pesquisa e de extensão.

Além disso, durante as ligações, foi possível orientar o grupo acerca do isolamento social, sanar dúvidas e desconstruir mitos em relação à COVID-19, que muitos traziam consigo. A preocupação dos idosos por serem considerados grupo de risco, com medo de desenvolverem a forma grave da doença, também foi foco de educação em saúde da equipe.

\section{Limitações do relato de experiência}

O grupo de idosos que participam do centro de convivência é heterogêneo quanto aos aspectos sociais, econômicos e culturais. Assim sendo, cada participante possui sua especificidade quanto ao acesso às tecnologias que foram utilizadas, principalmente para a distribuição das apostilas e vídeos. Desta forma, parte dos idosos não tiveram acesso ao material, impossibilitando-os de realizar os exercícios.

Considerando-se o momento atual de enfrentamento, o acompanhamento a distância esteve focado em idosos que possuem alguma forma de tecnologia de informação e comunicação, com vista a promoção do cuidado ao idoso que enfrenta o distanciamento social e que por vezes mora sozinho, longe da família. Contudo, esse idoso também possui limitações culturais e/ou financeiras quanto ao uso de telefones celulares e/ ou smartphones. Além do fato de serem idosos ativos e que se deparam com o isolamento social necessário ao enfrentamento da pandemia.

\section{Contribuições para a Enfermagem}

Espera-se com esse estudo contribuir para a prática clínica à medida que se utilizam novas tecnologias para promover o cuidado da pessoa idosa em Centros de Convivência. Assim, torna-se de extrema relevância os movimentos de reinvenção e readaptação dos profissionais de saúde durante a pandemia para manutenção da promoção da saúde do idoso.

Esse estudo ratifica a importância da manutenção do vínculo com o idoso e família durante o distanciamento social. Juntamente a isso, espera-se com este estudo promover a atuação da equipe interdisciplinar no cuidado à pessoa idosa explorando-se as múltiplas realidades relacionadas ao envelhecimento.

\section{CONSIDERAÇÕES FINAIS}

O acompanhamento a distância para idosos de centros de convivência pode ser considerado como uma estratégia dos profissionais da saúde nesse momento de pandemia, em que existe necessidade de readaptação para continuidade do cuidado. Além disso, o acompanhamento remoto nos centros de convivência permite a manutenção da interação com o idoso, reduzindo chances de evasão ao fim do isolamento social.

Ademais, cabe ressaltar o desenvolvimento da teleconsulta de enfermagem, com implementação das etapas do Processo de Enfermagem, essenciais no cotidiano do enfermeiro. Assim como o desenvolvimento de parcerias multidisciplinares com a equipe multiprofissional, o que possibilitou um olhar integral especializado à saúde da pessoa idosa.

Planejar, programar e avaliar atividades contribui para a formação de profissionais com bases consolidadas de cuidado gerontológico. Assim, as estratégias de ações extensionistas para acompanhamento a distância nos centros de convivência durante a pandemia, beneficiam o coletivo: sociedade, acadêmicos e docentes, promovendo a autonomia, independência, e consequente melhoria da qualidade de vida dos idosos. 


\section{FOMENTO}

Estudo parte do projeto de pesquisa "Sistematização da Assistência de Enfermagem, Teorias e Tecnologias nos Diferentes Grupos Populacionais", realizado com o apoio do Conselho Nacional de Desenvolvimento Científico e Tecnológico - CNPq.

\section{AGRADECIMENTO}

Agradecimentos: ao Centro Multidisciplinar de Pesquisa e Extensão sobre o Envelhecimento (CEMPE) Programa Renascer, da Universidade Federal do Estado do Rio de Janeiro; E ao Departamento Científico de Enfermagem Gerontológica da ABEn Nacional.

\section{REFERÊNCIAS}

1. Organização Mundial da Saúde (OMS). Novel Coronavirus (2019-nCoV) technical guidance, 2020. Disponível em: https:// www.who.int/health-topics/coronavirus\#tab=tab_1.

2. Brasil. Ministério da Saúde. Secretaria de Vigilância em Saúde. Boletim Epidemiológico. Infecção Humana pelo Novo Coronavírus (2019-nCoV). 2020. Disponível em: https://www.saude.gov.br/images/pdf/2020/fevereiro/04/ BoletimepidemiologicoSVS-04fev20.pdf

3. Hammerschmidt KSA, Santana RF. Saúde do idoso em tempos de pandemia Covid-19. Cogitare enferm, 2020; 25: e72846. doi: http://dx.doi.org/10.5380/ce.v25i0.DOI

4. Brasil. Ministério da Saúde. O que é o Coronavírus? (COVID-19). 2020. Disponível em: https://coronavirus.saude.gov.br/.

5. Brasil. Ministério da Cidadania. Estratégia Brasil Amigo da Pessoa Idosa. 2020. Disponível em: http://mds.gov.br/assuntos/ brasil-amigo-da-pessoa-idosa.

6. Argenta C, Nunes DN, Hammershmidt KSA, Niwa LMS. Souza PA, Nelo POC. Enfermagem gerontológica no cuidado ao idoso em tempos da covid-19. In: Associação Brasileira de Enfermagem; Santana RF, Castro FF, Freitas MC, Azevedo RCS, Alvares AM. Enfermagem gerontológica no cuidado do idoso em tempos da COVID 19. Brasília, DF: ABen/DCEG, 2020. $74 \mathrm{p}$.

7. Conselho Federal de Enfermagem (COFEN). Resolução COFEN n 634, de 26 de março de 2020. Autoriza e normatiza a teleconsulta de enfermagem como forma de combate à pandemia provocada pelo novo coronavírus (Sars-Cov-2). 2020. Disponível em: http://www.cofen.gov.br/resolucao-cofen-no-0634-2020_78344.html

8. Amaral A, Souza PA, Limeira A, Nascimento L, Castanheira A, Carneiro ML, et al. Apostila de exercícios cognitivos. 2020. Disponível em: http://educapes.capes.gov.br/handle/capes/573021

9. Liga Acadêmica de Enfermagem em Gerontologia UNIRIO. Cuidados na quarentena: quais cuidados os idosos devem ter durante a pandemia? 2020. Youtube. Disponível em: https://www.youtube.com/watch?v=7tpL-NXu3z8

10. Conselho Federal de Enfermagem (COFEN). Resolução COFEN n 358, de 15 de outubro de 2009. Dispõe sobre a Sistematização da Assistência de Enfermagem e a implementação do Processo de Enfermagem em ambientes, públicos ou privados, em que ocorre o cuidado profissional de Enfermagem, e dá outras providências. 2009. Disponível em: http:// www.cofen.gov.br/resoluo-cofen-3582009_4384.html

11. Machado TMD, Santana RF, Vaqueiro RD, Santos CTB, Souza PA. Telephone follow-up of the elderly after cataract surgery. British Journal of Visual Impairment. 2020;38(2): 184-195. Doi: 10.1177/0264619619874825

12. Gois BI. Interdisciplinaridade na saúde: um instrumento para o sucesso. Revista Brasileira de Ciências em Saúde. 2017;1(1):40-4. Disponível em: https://periodicos.uesc.br/index.php/rebracisa/article/view/1426 